\title{
An inversion identified in acl1-1 mutant functions as an enhancer of the acl1-1 phenotype
}

\author{
Naoko Kamata* and Yoshibumi Komeda \\ Department of Biological Sciences, Graduate School of Science, the University \\ of Tokyo, 7-3-1 Hongo, Bunkyo-ku, Tokyo 113-0033, Japan
}

(Received 11 June 2008, accepted 12 August 2008)

\begin{abstract}
The Arabidopsis acaulis1-1 (acl1-1) mutant exhibits severe growth defects when grown at $22^{\circ} \mathrm{C}$. The leaves are tiny and curled and the inflorescence stems are short. We identified an inversion mutation in the original acl1-1 plants. The acl1-1 plants were crossed with Columbia wild-type, and the acl1-1 phenotype and the inversion were segregated in the F2 generation. Compared to the original acl1-1 plants with the inversion, the genuine acl1-1 plants without the inversion grew larger and their inflorescence stems grew longer at $22^{\circ} \mathrm{C}$. When the plants were grown at $24^{\circ} \mathrm{C}$, the differences in growth became more apparent. We investigated the expression of genes located in the inversion. Two genes that were located at each end of the inversion were disrupted, and full-length transcripts were not expressed. Expressions of some genes within and adjacent to the inversion were also altered. Our results indicate that the expression of multiple genes may be involved in the enhancement of the acl1-1 phenotype.
\end{abstract}

Key words: acaulis1-1 (acl1-1), Arabidopsis thaliana, inversion, enhancer

\section{INTRODUCTION}

The Arabidopsis acaulis1 (acl1) mutant has defects in inflorescence elongation as represented in its name: no (a-) stem (caulis) (Tsukaya et al., 1993). Two acl1 mutant alleles were isolated in previous studies (Tsukaya et al., 1993). The acl1-1 mutation was induced by X-ray irradiation and the acl1-3 mutation by ethyl methanesulfonate (EMS) treatment. The acl1-1 plants exhibit severe phenotypes and develop tiny rosettes with curly leaves. The inflorescence stems are so short that only the upper parts of the stems can be seen over the rosette leaves. At the top of the inflorescence, a few fertile flowers are differentiated. The reduction in the size of rosettes and the length of the inflorescence stems are the common phenotype of acl1 mutants; however, the growth defects in the acl1-3 plants are more moderate. These growth defects observed in acl1 mutants are similar to the dwarf phenotypes of phytohormone mutants. Actually, treatments with common phytohormones such as auxin, brassinosteroid, and gibberellin failed to rescue acl phenotypes. The restoration of the acl1 phenotype occurs when acl1 plants are grown at higher temperatures, where acl1 plants became indistinguishable from

Edited by Minoru Murata

* Corresponding author. E-mail: kamatan@biol.s.u-tokyo.ac.jp Note: Supplementary materials in this article are at http:// wwwsoc.nii.ac.jp/gsj3/sup/83(4)Kamata/ wild-type plants. Ambient temperature is known to influence aspects of the appearances of plants, such as leaf size and stem length (Thingnaes et al., 2003, Atkin et al., 2006). However, the dramatic alteration in morphology of the acl1 phenotype at high temperature cannot be explained by general developmental variations controlled by ambient temperature. This unique phenomenon observed in acl1 mutants suggests that the ACL1 gene is novel gene controlling plant morphology at different ambient temperatures.

The cloning of the ACL1 gene will give us the answer why the acl1 plant morphology is dependent on temperature. During molecular mapping of acl1, we identified an inversion mutation in the original acl1-1 mutant. The acl1-1 phenotype was segregated from the inversion, indicating that the inversion was not the acl1 mutation itself. We obtained the genuine acl1-1 mutant without the inversion and, carried out thorough analyses of acl11 plants with and without the inversion. The inversion enhances the acl1-1 phenotype, inhibiting the elongation of inflorescence stems and reducing the size of rosettes. The inversion disrupts two genes located at each end of the inverted region. We analyzed the expression of these two genes and the genes within and adjacent to the inversion to understand the effects of the inversion. Our experimental data suggest that expression of multiple genes may be involved in the enhancement of the acl1-1 mutation. 


\section{MATERIALS AND METHODS}

Plant materials and growth conditions Arabidopsis thaliana ecotype Columbia (Col) was used as the wildtype. The original acl1-1 mutants were isolated as previously described (Tsukaya et al., 1993). Seeds were sown on water-moistened rock wool placed on vermiculite. For synchronous germination, seeds were placed in darkness at $4^{\circ} \mathrm{C}$ for 3 days before they were transferred to growth chambers. The growth chambers were set at 22 , 24,26 , or $28^{\circ} \mathrm{C}$ under long-day conditions ( $16 \mathrm{~h}$ light/ $8 \mathrm{~h}$ darkness). Plants were watered with medium containing $1.5 \mathrm{mM} \mathrm{NaH} \mathrm{PO}_{4} 2 \mathrm{H}_{2} \mathrm{O}, 0.26 \mathrm{mM} \mathrm{Na} \mathrm{HPO}_{4}, 3.0 \mathrm{mM}$ $\mathrm{KCl}, 0.0118 \mathrm{mM} \mathrm{Fe}$ (III)EDTA, $1.5 \mathrm{mM} \mathrm{MgSO}_{4} 7 \mathrm{H}_{2} \mathrm{O}, 5.0$ $\mathrm{mM} \mathrm{Ca}\left(\mathrm{NO}_{3}\right)_{2}, 0.5 \mathrm{mM} \mathrm{NH}_{4} \mathrm{Cl}, 3.0 \mu \mathrm{M} \mathrm{H}_{3} \mathrm{BO}_{4}, 0.1 \mu \mathrm{M}$ $\mathrm{ZnSO}_{4} 7 \mathrm{H}_{2} \mathrm{O}, 1 \mu \mathrm{M} \mathrm{CuSO}_{4} 5 \mathrm{H}_{2} \mathrm{O}, 10 \mu \mathrm{M} \mathrm{MnSO}_{4} 4 \mathrm{H}_{2} \mathrm{O}$, $0.175 \mu \mathrm{M} \mathrm{MoO}_{3}$, and $0.13 \mu \mathrm{M} \mathrm{CoCl}_{2} 6 \mathrm{H}_{2} \mathrm{O}$.

We obtained the transgenic line SGT754-5-3, which has a T-DNA insertion in the 3rd intron of At4g21960 (Landsberg erecta (L-er) background), and two other transgenic lines SALK_018861 and SALK_044071, which have TDNA inserted in the promoter region and 3'UTR of At4g22250, respectively (both are Col background, Alonso et al., 2004). The full length of At4g21960 transcript was undetectable in SGT754-5-3. Transcripts of At4g22250 in SALK_018861 and SALK_044071 were in detectable level in RT-PCR.

Examination of the inversion Genomic DNA was extracted from rosette leaves as described by Edwards et al. (1991). Primers used for the examination of the existence of genomic region from At4g22290 to At4g21690 were designed using sequences more than $50 \mathrm{bp}$ distant from the gene regions annotated by TAIR (http:// www.arabidopsis.org). Expected PCR fragments were less than $7 \mathrm{Kbp}$ in length, and were amplified with Ex taq (Takara Bio). PCR fragments were then treated with several restriction enzymes and cleaved into shorter fragments to identify the changes in fragment length more precisely by agarose gel electrophoresis. In the case of genes longer than $7 \mathrm{Kbp}$, genes were separated in two parts to ensure the effective PCR amplification. For the amplification of At4g21960, the following primer pair was used: NK215, 5'-GAGATCAGTAAAATAGATCG-3' and NK216, 5'-TTTAAGGAGCGTGCATTGC-3' were used as primers. Primers used to amplify At4g22250 were NK232: 5'TATAATGTCATCATCACTGC-3' and NK240, 5'-TCGAGTATCTCAATGATCGG-3'; and to amplify At4g21920, NK248, 5'-AAACATCAAACTTCACGGAG-3' and NK249, 5'AATACGTAGTTTTGACCTGG-3'. PCR fragments approximately $2.2 \mathrm{Kbp}, 0.8 \mathrm{Kbp}$ and $3.3 \mathrm{Kbp}$ in length were obtained by PCR for At4g21960, At4g22250, and At4g21920, respectively. The primers NK255, 5'AGATCACATTGAATCTGCAG-3' and NK256, 5'-TAAGTCAGTGTGGAACTAAG-3' were designed from sequences in the non-gene-coding region between At4g21960 and At4g21970 to obtain $1.7 \mathrm{Kbp}$ PCR products. In this study, this primer pair was used only for the positive control of PCR (Fig. 1). To detect the conjugated fragments of At4g21960 and At4g22250, NK215 and NK240 were used to obtain an approximately $1.3 \mathrm{Kbp}$ PCR fragment. NK216 and NK232 were used to obtain an approximately 1.6 Kbp PCR fragment only from the inversed DNA. Conjugated fragments were sequenced with the BigDye ${ }^{\circ}$ Terminator v.1.1 Cycle Sequencing kit (Applied Biosystems).

A CAPS marker, MASC04642, was designed from a sequence at the polymorphism site between Col and L-er ecotypes. PCR was performed with the primer pair MASC04642-1, 5'-ACAGTAATCGCCTACTTGG-3' and MASC04642-2, 5'-ATTGAAATGCTGGCATTGGG-3'． PCR products were treated with EcoT14I. Two fragments were obtained from Col genomic DNA (399 bp and $176 \mathrm{bp}$ ) and a 575bp fragment was obtained from L-er genomic DNA.

Expression analysis Plants were grown for 10 days on the medium described above, supplemented with $0.8 \%$ Bacto agar (BD) and $2 \%$ sucrose at $22^{\circ} \mathrm{C}$ under long-day conditions. RNA was extracted using a RNeasy® Plant Mini Kit (Qiagen). Extracted RNA was treated with Cloned DNaseI (Takara Bio). The reverse-transcriptase reaction was carried out with the Random 9 mers to synthesize cDNA from every type of transcript. Both the reverse transcriptase (RT) reaction and the polymerase chain reaction (PCR) were carried out using an RNA PCR kit (AMV) Ver. 3.0 (Takara Bio). Specific primers to amplify transcripts of At4g21960 were NK244, 5'ACTGCGCGGTGGAGTCATG-3' and NK856, 5'GTAGCATGTGAGGGACGTGG-3'; for At4g22250, NK398, 5' AGACGGAGATTCCAGGTTG-3' and NK239, 5'-TTAGAGTTTCCGTTACCGAG-3', and for $E F 1 \alpha$ (At5g60390, as a control), NK24, 5'-ACTTGCAGCTATGGGTAAAG-3' and NK25, 5'-CGAAAGTCTCATCATTTGGC-3'.

Observation of cellular structure and evaluation of cell length Stems of plants grown for 40 days after germination were fixed overnight in FAA [70\% ethanol:formaldehyde:acetic acid $=18: 1: 1(\mathrm{v} / \mathrm{v})]$ then dehydrated in ethanol series. Dehydrated samples were submerged in $50 \%(\mathrm{v} / \mathrm{v})$ Technovit 7100 resin in an ethanol for $1 \mathrm{~h}$ then embedded in $100 \%$ Technovit resin as described in the manual (Kluzer). Sections (5- $\mu \mathrm{m}$ thick) were stained with $0.05 \%$ toluidine blue $\mathrm{O}$ dissolved in $1 \%(\mathrm{w} / \mathrm{v})$ $\mathrm{Na}_{2} \mathrm{BO}_{4} \mathrm{O}_{7} 10 \mathrm{H}_{2} \mathrm{O}$. Cell size was measured on digital camera-photographed images using image analysis software (Adobe Photoshop). The average cell length was calculated within individuals and then average and standard deviation of each strain was determined (Fig. 3). Two individuals were analyzed for the type 2 
metamer (Schultz and Haughn, 1991) of the Col $+/+$ plants at $22^{\circ} \mathrm{C}$, the type 2 metamer of the $\mathrm{Col}+/+$ plants at $24^{\circ} \mathrm{C}$, the type 2 metamer of the $\mathrm{Col}+/+$ plants at $28^{\circ} \mathrm{C}$, the type 3 metamer of the Col inv/inv plants at $28^{\circ} \mathrm{C}$, and the acl1-1 inv/inv plants at $22^{\circ} \mathrm{C}$. Five acl1$1+/+$ plants grown at $22^{\circ} \mathrm{C}$ were analyzed and four acl1$1+/+$ plants grown $24^{\circ} \mathrm{C}$. Three individuals from each strain and each temperature were used for all other analysis. Ten cells from each individual were assayed to evaluate t-value (Table 3 ).

\section{RESULTS}

Inversion mutation in the original acl1-1 Previous research showed that the acl1-1 mutation was mapped on chromosome 4 near AGAMOUS (AG) (Tsukaya et al., 1993). Our recent result from genetic mapping positioned the acl1-1 mutation between the CAPS marker G4539 and the polymorphism MASC04642 on chromosome 4. The acl1-1 mutant was induced by X-ray irradiation and exhibits a more severe phenotype than the EMS-mutagenized acl1-3 mutant. Thus, we suspected that the chromosomes of the acl1-1 mutant are seriously disrupted. In order to explore chromosome disruptions, approximately $260 \mathrm{Kbp}$ of the genomic region from At4g22290, the nearest gene to MASC04642, to At4g21690 was examined by amplifying annotated gene regions by PCR. As the result, two PCR products including At4g21960, which encodes a peroxidase (Apel and Hirt, 2004, Welinder et al., 2002), and At4g22250, which encodes a zinc finger protein (Kosarev et al., 2002), were absent in the original acl1-1 mutant (Fig. 1, AB). The other PCR products detected had lengths corresponding to those of the wild-type (data not shown). Every PCR product was present in the acl1-3 mutant, which indicated that the absence of the two PCR products was specific to the acl1-1 mutant. The two genes are located at a distance of approximately $120 \mathrm{Kbp}$ on the same chromosome, and the genomic regions between them were intact. One possibility was that the genomic region between At4g21960 and At4g22250 was inverted. To investigate this possibility, PCR experiments were performed using combinations of primers at At4g21960 and At4g22250. If these two genes are fused to each other, conjugated fragments would be detected. Both combinations of primers examined were successful in amplifying novel DNA fragments from the acl1-1 plants (Fig. 1C, see Fig. 1D for locations of primers). The conjugated fragments were cloned and their sequences confirmed that At4g21960 and At4g22250 were cleaved and fused each other (Fig. 1D). These results suggested that the $120 \mathrm{Kbp}$ region between At4g21960 and At4g22250 was inverted in the original acl1-1 mutant.

We crossed the original acl1-1 plants to Col wild-type to evaluate the segregation of the acl1-1 phenotype and the inversion. The F2 population was segregated into 496 (73.4\%) wild-type plants and 180 (26.6\%) acl1-1 plants at a $3: 1$ ratio $\left(\chi^{2}=0.716\right)$. We extracted DNA from 371 wild-type plants and 146 acl1-1 plants, and examined whether these plants carried the inversion (Table 1). Regarding the inversion, the segregation at $+/+$ : inv/+: inv/inv at the ratio of 1:2:1 was less reliable $\left(\chi^{2}\right.$ $\left.0.025(2)=7.38<\chi^{2}=9.07<\chi^{2} 0.010(2)=9.21\right)$. The acl1-1 phenotype did not necessarily cosegregate with the inversion. Thus, the inversion is independent of the cause of the acl1-1 phenotype. The recombination rate between the acl1-1 phenotype and the inversion was estimated to be $15.4 \%$ according to the segregation of the F2 plants. To simplify descriptions, we refer to the newly isolated acl1-1 plants without the inversion as acl1-1 +/+, the original acl1-1 plants with the inversion as acl1-1 inv/inv, wild-type plants as $\mathrm{Col}+/+$, and the wild-type phenotype (ACL1 / ACL1) plants with the inversion as Col inv/inv.

Full-length transcripts of At4g21960 and At4g22250 were not detected in the inv/inv plants (Fig. 1E). We cloned At4g21960 and At4g22250 from the acl1-3 plants and found that there was no mutation. Moreover, the expression levels of these two genes in the acl1-3 mutants were identical to those in the wild-type (data not shown). Thus, the acl1-3 phenotype does not require a mutation in At4g21960 nor At4g22250. T-DNA insertion lines in At4g21960 and At4g22250 had no obvious growth defects (data not shown). Together our results indicated that the loss of either of At4g21960 or At4g22250 does not alter morphology of the plants.

Expressions of genes related to the inversion We investigated the expression of two genes that are located at the fusion-points of in the inversion, At4g21960 and At4g22250. And the expressions of those genes located within and adjacent to the inversion were examined (Supplementary Fig. 1. also see Supplementary Table 1 for gene functions and sequences of primers used for analyses). Expression of some genes differed among the four plant strains: $\mathrm{Col}+/+, \mathrm{Col}$ inv/inv, acl1-1 +/+, and acl11 inv/inv. From RT-PCR experiments, we observed a decrease in expression of At4g22270 (IMMUTANS, Wu et al., 1999), an increase in At4g22214 (defensin like protein, Silverstein et al., 2005), and a slight increase in At4g22235 (defensin like protein) in two inv/inv plants. In the acl1-1 inv/inv plants, we found increased expression of At4g22050 (aspartyl protease family protein) and At4g22070 (WRKY DNA-BINDING PROTEIN 31, Eulgem et al., 2000) and decreased expression of At4g21990 (APS REDUCTASE 3, Houston et al., 2005), At4g22010 (SKU5 SIMILAR 4, Sedbrook et al., 2002) and At4g22212 (defensin like protein). Among the genes investigated, At4g22080 (pectate lyase family protein), At4g22090 (pectate lyase family protein), At4g22210 (Cys-rich protein), 


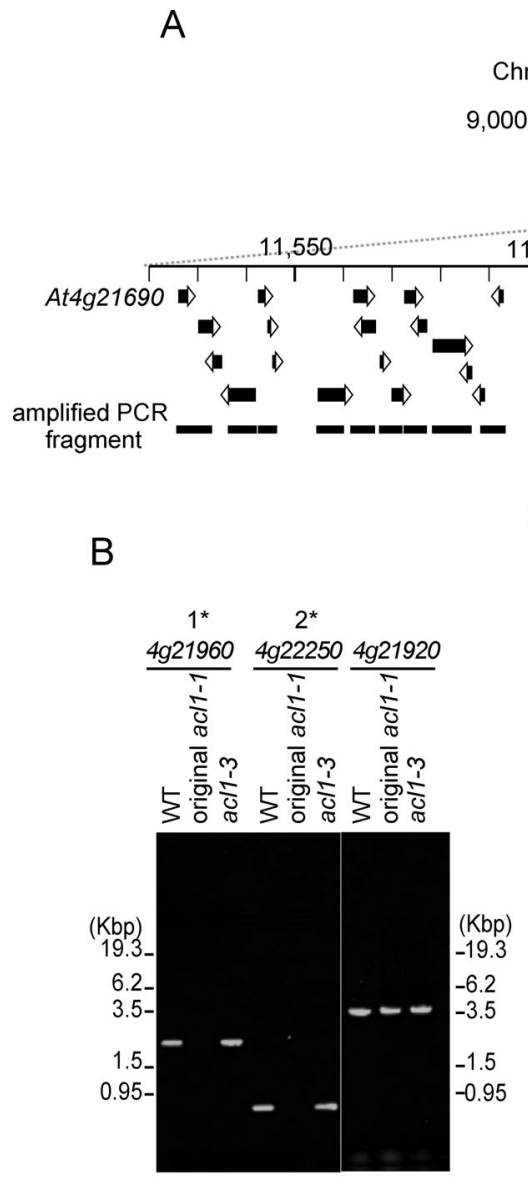

Chromosome 4 putative region including acl1-1.
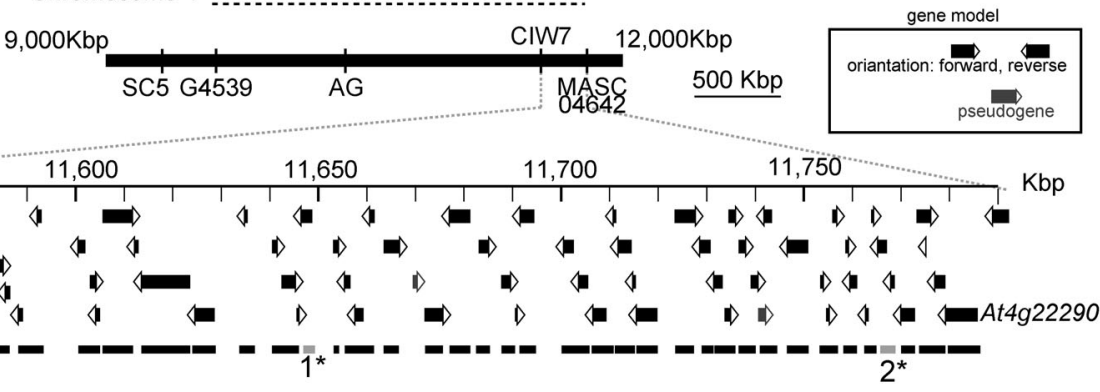

D WT

C

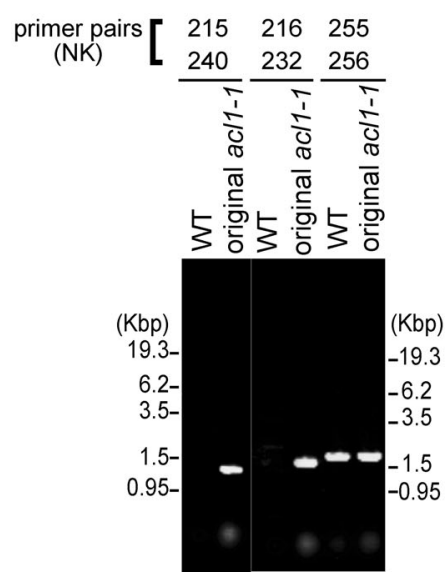

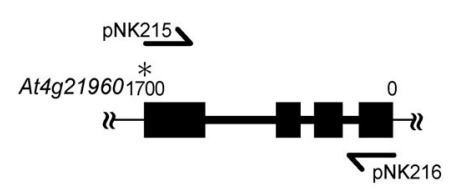

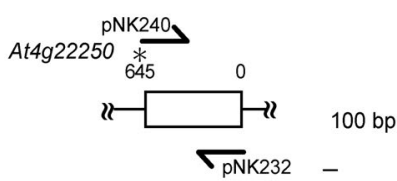

$\leftarrow 4 g 21950$

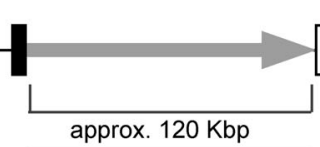

$4 g 22260 \rightarrow$

inversion in acl1-1

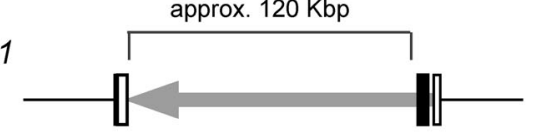

At4g21960*
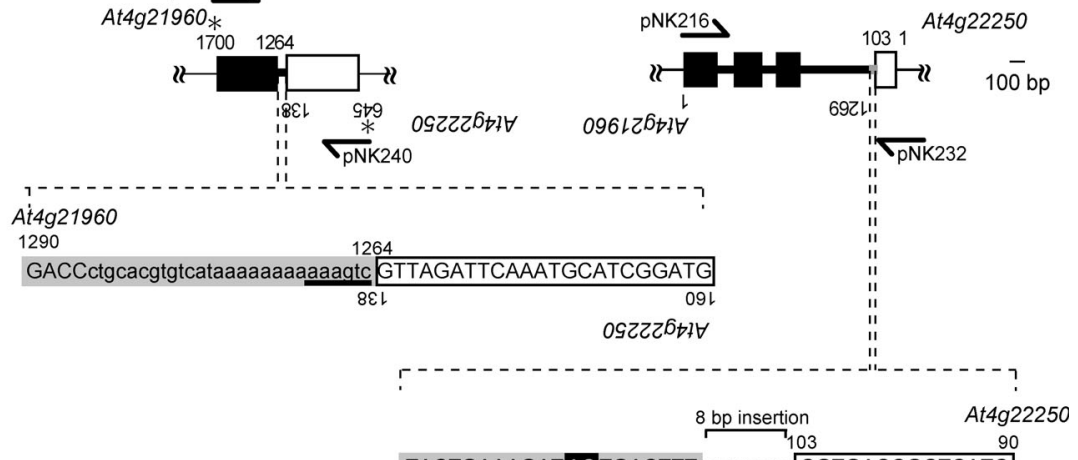
osz

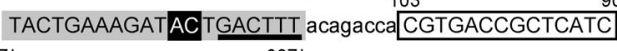
096เこ6+1

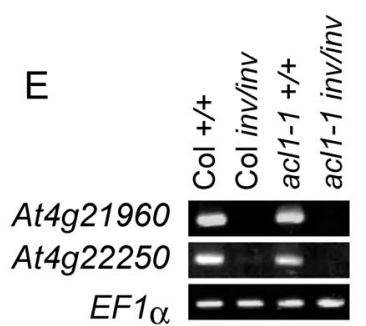

Fig. 1. Identification of inversion in the original acl1-1 mutants. A, Locations of genes assayed in this study. B, PCR performed with DNA from wild-type, the original acl1-1 mutant, and the acl1-3 mutant to detect full-length genomic regions of At4g21960 and At4g22250. C, Novel conjugated fragments detected in the original acl1-1 mutant. D, Structure of wild-type At4g21960 and At4g22250 and conjugated genes caused in the inversion. Filled and open squares: exons. Bold lines: introns. Arrows with pNKnumber: positions of primers used. Within nucleotide sequences, gray shading, At4g21960; white boxes, At4g22250; capital letters, exons; small letters, introns. Underlined 6 bp were duplicated. Black shading indicates 2 bp substitution from TT to AC. A 34-bp fragment was deleted from At4g22250. E, RT-PCR performed with RNA from 10-day-old plants. 
Table 1. Segregation of acl1-1 phenotype and the inversion

\begin{tabular}{lccc}
\hline \hline Phenotype & \multicolumn{3}{c}{ Inversion } \\
\cline { 2 - 4 } & $\begin{array}{c}\text { Wild-type } \\
(+/+)\end{array}$ & $\begin{array}{c}\text { Hemizygote } \\
(\text { inv/+) }\end{array}$ & $\begin{array}{c}\text { Homozygote } \\
(\text { inv/inv })\end{array}$ \\
\hline Wild-type & 123 & 203 & 45 \\
acl1-1 & 5 & 22 & 119 \\
\hline
\end{tabular}

At4g22217 (defensin like protein) and At4g22230 (defensin like protein) decreased in both the acl1-1 plants, regardless of the inversion. The expression of At4g22030 (F-box family protein) and At4g22100 (glycosyl hydrolase family 1 protein) was decreased in the Col inv/inv plants and the acl1-1 $+/+$ plants, and even further decreased in the acl1-1 inv/inv plants compared to the $\mathrm{Col}+/+$ plants. The expression patterns of these genes in the acl1-3 plants were similar to those of the $\mathrm{Col}+/+$ plants. We speculated that the alteration in the expression pattern is due to mutations in At4g22050, At4g22080, At4g22090, and At4g22270. Thus, these genes were cloned from acl1-1 plants and acl1-3 plants and sequenced. As the results, these genes were identical to the wild-type (data not shown). Furthermore, we found that expression of the partial transcripts from the fused fragments of both At4g21960 and At4g22250 was maintained in the inv/inv plants (Supplementary Fig. 1B). In the case of At4g22250, the partial transcripts were rather increased in the plants with the inversion. Novel transcripts were also detected by RT-PCR using combinations of primers at At4g21960 and At4g22250 specific to the plants with the inversion. Thus, the inversion affected expression of a wide range of genes.

The acl1-1 phenotype was enhanced by the inversion We compared the phenotype of acl1-1 plants without the inversion to acl1-1 plants with the inversion. At $22^{\circ} \mathrm{C}$, there were no apparent differences between $\mathrm{Col}$ inv/inv plants and $\mathrm{Col}+/+$ plants. The plant heights were identical to each other (Fig. 2A, Table 2). Both the acl1-1 +/+ plants and the acl1-1 inv/inv plants exhibited the acl1-1 phenotype with small curly leaves and short inflorescence stems. However, the comparison of the acl1-1 + /+ plants and the acl1-1 inv/inv plants made us realize the difference between these two genotypes. The rosettes of the acl1-1 inv/inv plants appeared slightly smaller than those of the acl1-1 $+/+$ plants (Fig. 2A). The height of the acl1-1 +/+ plants was significantly different to that of the acl1-1 inv/inv plants, indicating that the growth defects of the acl1-1 mutants are enhanced by the inversion (Table 2 ).

We observed sections of inflorescence stems, and found that the acl1-1 mutation caused serious defects in the length of cells. The reduction of the cell length was significant in acl1-1 background in all types of tissues examined; cells in the epidermis, the outermost layer of cortex, and the pith, (Table 3). The reduction in the size of the epidermal cells was most severe, and the cells in pith also were significantly affected by the acl1-1 mutation. The

Table 2. Measurement of plant height at $22^{\circ} \mathrm{C}$

\begin{tabular}{lrcrl}
\hline \hline Genotype & \multicolumn{3}{c}{ Average \pm standard deviation $(\mathrm{cm})$} & $\mathrm{t}$-value \\
\hline acl1-1+/+* & 6.0 & \pm 1.8 & $(\mathrm{n}=39)$ & \\
acl1-1 inv/inv* & 4.3 & \pm 1.2 & $(\mathrm{n}=31)$ & $\mathrm{t}=4.52^{\dagger}$ \\
Col $+/+^{* *}$ & 179.7 & \pm 17.4 & $(\mathrm{n}=16)$ & \\
Col inv/inv * $^{*}$ & 180.0 & \pm 29.1 & $(\mathrm{n}=7)$ & $\mathrm{t}=0.031^{\dagger \dagger}$
\end{tabular}

Plants were grown for 40 days.

* Total plant height including the rosette and the inflorescence stem.

** Length of the inflorescence stem was measured as plant height.

${ }^{\dagger}$ Difference between acl1-1 $+/+$ and acl1-1 inv/inv is significant (significance level: $\alpha=0.05$ ).

${ }^{+} \mathrm{Col}+/+$ and Col inv/inv are identical (significance level: $\alpha=$ 0.05).
$\mathrm{A}, 22^{\circ} \mathrm{C}$

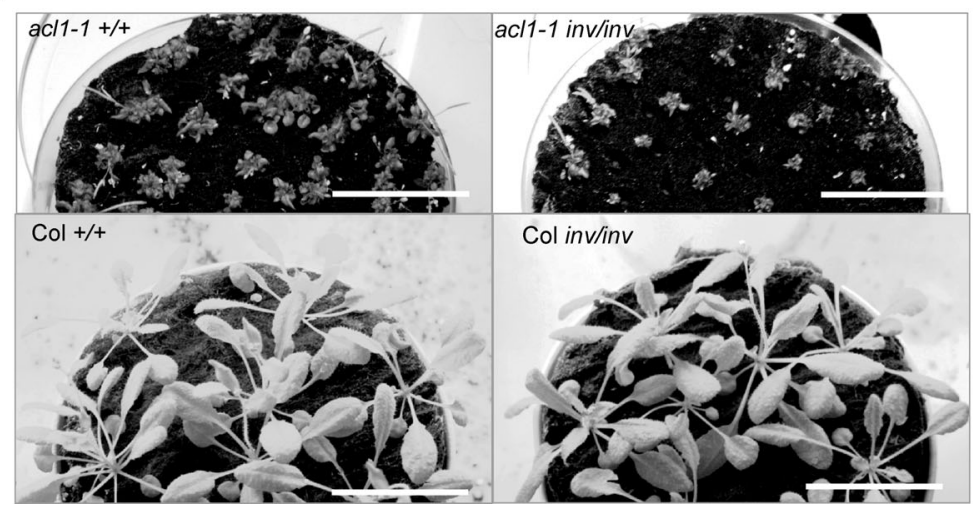

$B, 24^{\circ} \mathrm{C}$

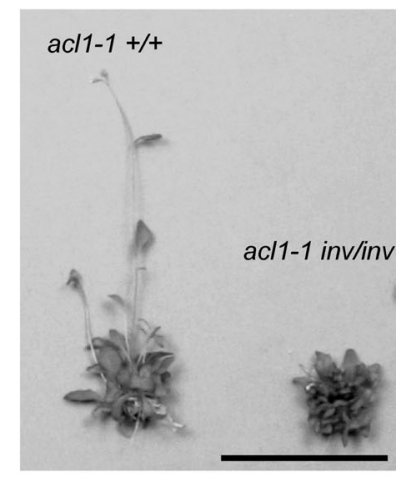

Fig. 2. Enhancement of acl1-1 phenotype by the inversion. A, Overall development of $a c l 1-1+/+$ plants and $a c l 1-1$ inv $/$ inv plants was observed 40 days after germination. Rosettes of $\mathrm{Col}+/+$ plants and Col inv/inv plants were observed 35 days after germination. Plants were grown at $22^{\circ} \mathrm{C}$. Bar $=3 \mathrm{~cm}$. B, Plants were grown at $24^{\circ} \mathrm{C}$ for 40 days after germination. Bar $=3 \mathrm{~cm}$. 
Table 3. Comparison between cell length of $+/+$ and inv/inv plants

\begin{tabular}{|c|c|c|c|c|c|}
\hline & & $\begin{array}{l}\text { Epidermis } \\
(\mu \mathrm{m})\end{array}$ & $\begin{array}{c}\text { Cortex } \\
(\mu \mathrm{m})\end{array}$ & $\begin{array}{l}\text { Pith } \\
(\mu \mathrm{m})\end{array}$ & $\mathrm{n}^{*}$ \\
\hline $22^{\circ} \mathrm{C}$ acl1-1 +/+ & & $43.0 \pm 14.6$ & $28.5 \pm 6.38$ & $78.0 \pm 25.0$ & 50 \\
\hline \multirow{2}{*}{$22^{\circ} \mathrm{C}$ acl1-1 inv/inv } & & $21.2 \pm 9.25$ & $26.1 \pm 6.84$ & $43.6 \pm 11.1$ & 20 \\
\hline & t-value & $6.19^{\dagger}$ & 1.36 & $5.91^{\dagger}$ & \\
\hline $22^{\circ} \mathrm{C} \mathrm{Col}+/+2 \mathrm{met}^{* *}$ & & $266.7 \pm 100.4$ & $40.2 \pm 8.61$ & $175.3 \pm 48.9$ & 20 \\
\hline \multirow[t]{2}{*}{$22^{\circ} \mathrm{C} \mathrm{Col} \mathrm{inv/inv} 2 \mathrm{met}^{* *}$} & & $243.2 \pm 54.0$ & $38.5 \pm 11.9$ & $197.2 \pm 39.1$ & 30 \\
\hline & t-value & 1.07 & 0.552 & -1.75 & \\
\hline $22^{\circ} \mathrm{C} \mathrm{Col}+/+3 \mathrm{met}^{* *}$ & & $232.1 \pm 54.4$ & $31.2 \pm 9.03$ & $178.1 \pm 54.6$ & 30 \\
\hline \multirow[t]{2}{*}{$22^{\circ} \mathrm{C} \mathrm{Col} \mathrm{inv/inv} \mathrm{3met**}$} & & $245.9 \pm 56.5$ & $31.7 \pm 7.96$ & $159.8 \pm 43.7$ & 20 \\
\hline & t-value & -0.803 & -0.209 & 1.25 & \\
\hline $24^{\circ} \mathrm{C}$ acl1-1 $+/+2$ met $^{* *}$ & & $112.6 \pm 62.3$ & $28.6 \pm 8.71$ & $92.7 \pm 35.5$ & 40 \\
\hline \multirow[t]{2}{*}{$24^{\circ} \mathrm{C}$ acl1-1 inv /inv $2 \mathrm{met}^{* *}$} & & $25.6 \pm 7.24$ & $36.4 \pm 10.9$ & $72.1 \pm 39.3$ & 20 \\
\hline & t-value & $6.20^{\dagger}$ & $-2.99^{\dagger}$ & $2.04^{\dagger}$ & \\
\hline $24^{\circ} \mathrm{C}$ acl1-1 $+/+3$ met $^{* *}$ & & $205.0 \pm 83.1$ & $31.5 \pm 9.56$ & $128.1 \pm 31.2$ & 30 \\
\hline \multirow[t]{2}{*}{$24^{\circ} \mathrm{C}$ acl1-1 inv /inv 3met** } & & $23.2 \pm 14.4$ & $13.7 \pm 6.30$ & $37.4 \pm 18.6$ & 30 \\
\hline & t-value & $11.81^{\dagger}$ & $8.53^{\dagger}$ & $13.67^{\dagger}$ & \\
\hline
\end{tabular}

${ }^{\dagger}$ Difference is significant (significance level: $\alpha=0.05$ ).

* Total number of cells assayed for calculate t-value.

** Metameric type of apical meristems: 2 met, the type 2 metamer, main inflorescence stem differentiates cauline leaves with elongating internodes; 3met, the type 3 metamer, main florescence stem bears flowers without bracts formed upon the type 2 metamer (Schultz and Haughn, 1991).
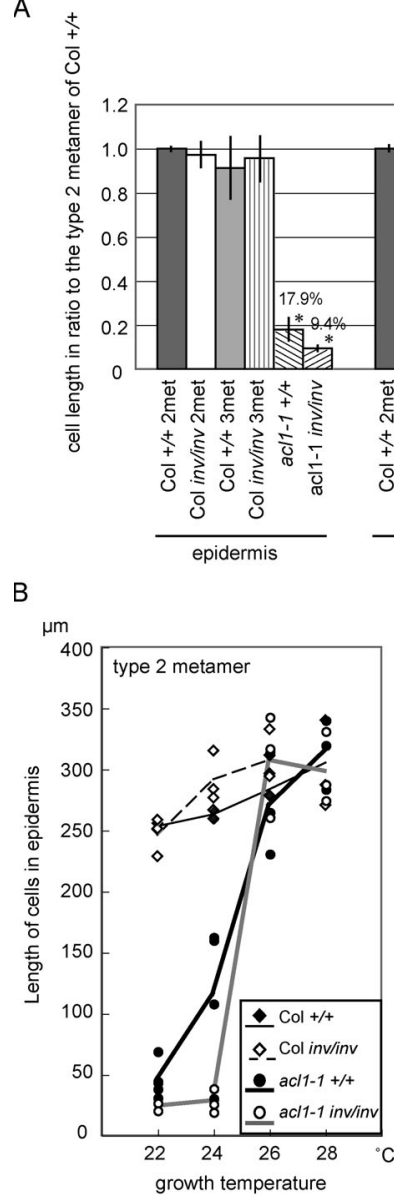

C $\mu \mathrm{m}$

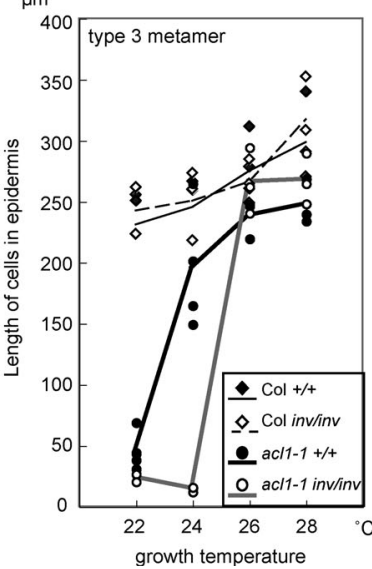

acl1-1 inv/inv plants exhibited more severe reduction in the cell length than the acl1-1+/+ plants. Unlike cells in epidermis and pith, the length of cortex cells was less affected. In addition to the severe reduction in length the differentiation of cells appeared to be inhibited in the inflorescence stems of the acl1-1+/+ plants and the acl11 inv/inv plants.

The inversion affects acl1-1 growth at $24^{\circ} \mathrm{C}$ We observed morphological characteristics under several different temperature conditions. Both the acl1-1+/+ plants and the acl1-1 inv/inv plants were able to restore their plant morphology when grown at $28^{\circ} \mathrm{C}$ as previously reported (Tsukaya et al., 1993). Plants tend to become more slender at higher temperatures, as the result of the general effects of higher temperature. These general effects of higher temperature were observed in the Col inv/inv plants as well as in the Col $+/+$ plants throughout our experiments. Correspondingly, the cells became longer at higher temperature (Fig. 3, B and C). Plants were

Fig. 3. Length of cells of inflorescence stems. A, Lengths of cells in epidermis, cortex, and pith were measured and compared to the type 2 metamer of $\mathrm{Col}+/+$ plants. Percentages indicate the ratio to the length of type 2 metamer of the Col $+/+$ plants. 2 met $=$ type 2 metamer, 3 met $=$ type 3 metamer. $B, C$, Length of cells in epidermis of type 2 (B) and type $3(\mathrm{C})$ metamer at $22,24,26$, and $28^{\circ} \mathrm{C}$. At $22^{\circ} \mathrm{C}$, the type 2 and type 3 metamers of the acl1-1+/+ plants and the acl1-1 inv/inv plants were assayed together. Lines: averages of each plant strain. 
Table 4. Measurement of plant height at $26^{\circ} \mathrm{C}$

\begin{tabular}{|c|c|c|c|c|}
\hline Genotype & Avera & standa & tion $(\mathrm{cm})$ & t-value \\
\hline $\operatorname{acl} 1-1+/+*$ & 227.6 & \pm 27.5 & $(\mathrm{n}=12)$ & $\mathrm{t}=-0.350^{\dagger}$ \\
\hline acl1-1 inv/inv* & 227.3 & \pm 52.1 & $(\mathrm{n}=8)$ & $\mathrm{t}=-0.242^{\dagger}$ \\
\hline $\mathrm{Col}+/+^{*}$ & 221.4 & \pm 54.5 & $(\mathrm{n}=12)$ & \\
\hline Col inv/inv* & 231.0 & \pm 58.7 & $(\mathrm{n}=16)$ & $\mathrm{t}=-0.519^{\dagger}$ \\
\hline
\end{tabular}

Plants were grown for 40 days.

* Length of the inflorescence stem was measured as plant height.

${ }^{\dagger}$ Height of the plants is identical to $\mathrm{Col}+/+$.

grown at 24 and $26^{\circ} \mathrm{C}$ to observe morphology at the intermediate temperatures. The restoration of the acl1-1 phenotype to wild-type was not complete at $24^{\circ} \mathrm{C}$ (Fig. 2B). However, the difference between the acl1-1 $+/+$ plants and the acl1-1 inv / inv plants became more obvious at $24^{\circ} \mathrm{C}$. The inflorescence stems of acl1-1+/+ plants elongated to approximately $5 \mathrm{~cm}$ in length (average \pm standard deviation, $5.2 \pm 3.4 \mathrm{~cm}, \mathrm{n}=12$ ). On the other hand, the inflorescence stems of acl1-1 inv/inv were as short as the plants grown at $22^{\circ} \mathrm{C}$. The length of cells, including those in the cortex, became significantly different between the acl1-1+/+ plants and the acl1-1 inv/inv plants (Table 3). Neither the acl1-1+/+ plants nor the acl1-1 inv /inv plants fully restored the acl1-1 phenotype at $24^{\circ} \mathrm{C}$, however the inflorescence stems of both the acl1$1+/+$ plants and the acl1-1 inv/inv plants elongated to a similar length to those of Col plants at $26^{\circ} \mathrm{C}$. Complete restoration to wild-type occurred at $26^{\circ} \mathrm{C}$, as measured by the length of the epidermal cells in the inflorescence stems (Table 4, Fig. 3, B and C). There was no difference between the acl1-1+/+ plants and the acl1-1 inv/inv plants at temperatures exceeding $26^{\circ} \mathrm{C}(\mathrm{t}=0.014$ for plant height at $26^{\circ} \mathrm{C}$ ).

\section{DISCUSSION}

In this study, we isolated the "genuine" acl1-1 plants without the inversion. By comparing these plants to the original acl1-1 plants with the inversion, we showed that the inversion functions as an enhancer of the acl1-1 phenotype.

In the previous study, it was reported that the length of the inflorescence stems of acl1-1 plants varied among plants, even though they were grown side by side under the same conditions at $22^{\circ} \mathrm{C}$ (Tsukaya et al., 1993). We suggest that this unknown factor that influenced the growth of the original acl1-1 mutants is the inversion located on chromosome 4. The effects of the inversion on the growth of acl1-1 plants were evident at $22^{\circ} \mathrm{C}$. The height of plants, the size of rosettes, and the length of cells were remarkably reduced in the acl1-1 inv/inv plants compared with the acl1-1+/+ plants (Tables 2 and 3 , Figs. $2 \mathrm{~A}$ and $3 \mathrm{~A}$ ), thus, the inversion enhances the acl11 phenotype. The inversion functioned more effectively as an enhancer of the acl1-1 phenotype when plants were grown at $24^{\circ} \mathrm{C}$. The acl1-1 phenotype is gradually restored to wild-type as temperature increases. The acl1-1 $+/+$ plants partially restored the mutant phenotype to wild-type but the acl1-1 inv/inv plants did not (Fig. 2B). When the mutant phenotype was fully restored to wild-type in the acl1-1 plants at $26^{\circ} \mathrm{C}$ or $28^{\circ} \mathrm{C}$, the difference between the acl1-1+/+ plants and the acl11 inv/inv plants disappeared. The inversion itself was not sufficient to alter plant morphology in the wild-type background. It appears that the inversion enhances the defects in the plant growth only when growth defects are already present in acl1-1 plants.

Many aspects of plant development are affected in acl11 plants. We analyzed expressions of genes within and adjacent to the inversion. In addition to loss of fulllength transcripts of At4g21960 and At4g22250, the expression patterns of some other genes were altered (Supplementary Fig. 1). There were several genes that showed remarkable alteration in expression pattern depending on the inversion (e.g. At4g22270, At4g22214, and At4g22235). As the inversion affected the expression of many genes, a complex effect caused by multiple genes may be responsible for the enhancement of the acl1-1 phenotype. At present, we only analyzed the expression of genes within and adjacent to the inversion using RNA extracted from young seedlings. However, organ-specific and/or age-specific expression of these genes may also be affected by the inversion. To further clarify why the acl1-1 phenotype is enhanced by the inversion, we will further investigate genes involved in the inversion and their relationship to the acl1-1 phenotype.

The original acl1-1 mutant was obtained by X-ray irradiation. In most higher plants, including Arabidopsis, DNA double-strand breaks caused by ionizing irradiation (fast neutron, X-ray, and $\gamma$-ray) are predominantly repaired by non-homologous end-joining rather than by simple ligation or accurate homologous recombination. In non-homologous end-joining, any end can fuse with any end. Thus, the repair of double-strand breaks in plants is suggested to be error-prone (Gorbunova and Levy, 1999). Sometimes, the repair of double-strands breaks have a very complex DNA rearrangements, combining deletions, insertions, inversions and duplications of the original sequence (Shirley et al., 1992). The inversion isolated from the original acl1-1 was also complex at the border regions, including deletions, insertions, duplications, and substitutions (Fig. 1D). The acl1-1 mutation itself would be caused by the error in the repair of doublestrand breaks caused by X-ray irradiation. The "genuine" acl1-1 plants still exhibit more severe growth defects than the acl1-3 plants induced by EMS (data not shown), which induces base substitutions from G:C to A:T (Colbert et al., 2001). Considering the differences in mutagens 
and phenotypes between acl1-1 and acl1-3 plants, the acl1-1 mutation is expected to be a serious DNA rearrangement rather than a simple base substitution.

We are continuing our molecular mapping of acl1-1 and, in the near future, the details of the acl1-1 mutation will be revealed.

We acknowledge the Institute of Molecular Agrobiology, National University of Singapore for providing the seed line SGT754-5-3 and ABRC (Arabidopsis Biological Resource Center) for providing SALK_018861 and SALK_044071. This work was supported by a Grant-in-Aid to N. K. for Japan Society of the Promotion Science (JSPS) Fellows (No. 2010890).

\section{REFERENCES}

Alonso, J. M., Stepanova, A. N., Leisse, T. J., Kim, C. J., Chen, H., Shinn, P., Stevenson, D. K., Zimmerman, J., Barajas, P., Cheuk, R. et al., (2003) Genome-wide insertional mutagenesis of Arabidopsis thaliana. Science 301, 653-657.

Apel, K., and Hirt, H. (2004) REACTIVE OXYGEN SPECIES: Metabolism, oxidative stress, and signal transduction. Annu. Rev. Plant Biol. 55, 373-399.

Atkin, O. K., Loveys, B. R., Atkinson, L. J., and Pons, T. L. (2006) Phenotypic plasticity and growth temperature: understanding interspecific variability. J. Exp. Bot. 57, 267-281.

Colbert, T., Till, B. J., Tompa, R., Reynolds, S., Steine, M. N., Yeung, A. T., McCallum, C. M., Comai, L., and Heinkoff, S. (2001) High-throughput screening for induced point mutations. Plant Physiol. 126, 480-484.

Edwards, K., Johnstone, C., and Thompson, C. (1991) A simple and rapid method for the preparation of plant genomic DNA for PCR analysis. Nucleic Acids Res. 19, 1349-1350.

Eulgem, T., Rushton, P. J., Robatzek, S., and Somssich, I. E. (2000) The WRKY superfamily of plant transcription factors. Trends Plant Sci. 5, 199-206.

Gorbunova, V., and Levy, A. A. (1999) How plants make ends meet: DNA double-strand break repair. Trends Plant Sci. 4, 263-269.

Houston, N. L., Fan, C., Xiang, J. Q., Schulze, J. M., Jung, R., and Boston, R. S. (2005) Phylogenetic analyses identify 10 classes of the protein disulfide isomerase family in plants, including single-domain protein disulfide isomerase-related proteins. Plant Physiol. 137, 762-778.

Kosarev, P., Mayer, K. X., and Hardtke, C. S. (2002) Evaluation and classification of RING-finger domains encoded by the Arabidopsis genome. Genome Biol. 3, research0016.10016.12

Sedbrook, J. C., Carroll, K. L., Hung, K. F., Masson, P. H., and Somerville, C. R. (2002) The Arabidopsis SKU5 gene encodes an extracellular glycosyl phosphatidylinositol-anchored glycoprotein involved in directional root growth. Plant Cell 14, 1635-1648.

Schultz, E. A., and Haughn, G. W. (1991) LEAFY, a homeotic gene that regulate inflorescence development in Arabidopsis. Plant Cell 3, 771-781.

Shirley, B. W., Hanley, S., and Goodman, H. M. (1992) Effects of ionizing radiation on a plant genome: analysis of two Arabidopsis transparent testa mutations. Plant Cell 4, 333-347.

Silverstein, K. A., Graham, M. A., Paape, T. D., and VandenBosch, K. A. (2005) Genome organization of more than 300 Defensin-Like genes in Arabidopsis. Plant Physiol. 138, 600-610.

Thingnaes, E., Torre, S., Ernstsen, A., and Moe, R. (2003) Day and night temperature responses in Arabidopsis: effects on gibberellin and auxin content, cell size, morphology and flowering time. Ann. Bot. 92, 601-612.

Tsukaya, H., Naito, S., Redei, G. P., and Komeda, Y. (1993) A new class of mutations in Arabidopsis thaliana, acaulis1, affecting the development of both inflorescences and leaves. Development 118, 751-764.

Welinder, K. G., Justesen, A. F., Kjærsgård, I. V. H., Jensen, R. B., Rasmussen, S. K., Hans, M., Jespersen, H. M., and Duroux, L. (2002) Structural diversity and transcription of class III peroxidases from Arabidopsis thaliana. Eur. J. Biochem. 269, 6063-6081.

Wu, D., Wright, D. A., Wetzela, C., Voytas, D. F., and Rodermel, S. (1999) The IMMUTANS variegation locus of Arabidopsis defines a mitochondrial alternative oxidase homology that functions during early chloroplast biogenesis. Plant Cell 11, 43-56. 